\title{
The role of the orbitofrontal cortex in human discrimination learning
}

\author{
Henry W. Chase ${ }^{\mathrm{a}, \mathrm{b}, *}$, Luke Clark ${ }^{\mathrm{a}, \mathrm{b}}$, Catherine E. Myers ${ }^{\mathrm{c}}$, Mark A. Gluck ${ }^{\mathrm{d}}$, \\ Barbara J. Sahakian ${ }^{\mathrm{a}, \mathrm{e}}$, Edward T. Bullmore ${ }^{\mathrm{a}, \mathrm{e}}$, Trevor W. Robbins ${ }^{\mathrm{a}, \mathrm{b}}$ \\ ${ }^{a}$ Behavioural and Clinical Neuroscience Institute, University of Cambridge, UK \\ ${ }^{\mathrm{b}}$ Department of Experimental Psychology, University of Cambridge, UK \\ ${ }^{\mathrm{c}}$ Department of Psychology, Rutgers University, USA \\ ${ }^{\mathrm{d}}$ Center for Molecular and Behavioral Neuroscience, Rutgers University, USA \\ ${ }^{\mathrm{e}}$ Department of Psychiatry, University of Cambridge School of Clinical Medicine, Addenbrooke's Hospital, Cambridge, UK
}

Received 17 March 2007; received in revised form 6 December 2007; accepted 9 December 2007

Available online 23 December 2007

\begin{abstract}
Several lines of evidence implicate the prefrontal cortex in learning but there is little evidence from studies of human lesion patients to demonstrate the critical role of this structure. To this end, we tested patients with lesions of the frontal lobe $(n=36)$ and healthy controls $(n=35)$ on two learning tasks: the weather prediction task (WPT), and an eight-pair concurrent visual discrimination task ('Choose'). Performance of both tasks was previously shown to be disrupted in patients with Parkinson's disease; the Choose deficit was only present when patients were medicated. Patients with damage to the orbitofrontal cortex (OFC) were significantly impaired on Choose, compared to both healthy controls and non-OFC lesion patients. The OFC lesion patients showed a mild deficit on the first 50 trials of the WPT, compared to the control subjects but not non-OFC lesion patients. The selective deficit in the OFC patients on Choose performance could not be attributed to the larger lesion size in this group, and the deficit was not correlated with the volume of damage to adjacent prefrontal subregions (e.g. anterior cingulate cortex). These data support the notion that the OFC play a role in normal discrimination learning, and suggest qualitative similarities in learning performance of patients with OFC damage and medicated PD patients.
\end{abstract}

(C) 2007 Elsevier Ltd. All rights reserved.

Keywords: Orbitofrontal; Prefrontal cortex; Learning; Discrimination

\section{Introduction}

The prefrontal cortex (PFC) has a well-established role in learning and memory, although the exact nature of this contribution is unclear. One account is that it contributes to executive or intentional aspects of learning, but not the habitual or incremental acquisition of information (e.g. Hay, Moscovitch, \& Levine, 2002). Other neurobiologically inspired dual system models of categorisation (e.g. Frank \& Claus, 2006; Maddox \& Ashby, 2004) relate the function of the frontal lobe in the flexible use of explicit, verbalisable rules or stimulus values. Lesion studies involving experimental animals have suggested that frontal lobe integrity is critical for intact performance on more com-

\footnotetext{
* Corresponding author at: Behavioural and Clinical Neuroscience Institute, University of Cambridge, Downing Street, Cambridge CB2 3EB, UK.

E-mail address: hc277@cam.ac.uk (H.W. Chase).
}

plex learning tasks: for example, conditional learning tasks, in which the subject must associate an arbitrary motor response with a stimulus to receive a reward, are generally more affected by damage to the frontal lobe than non-conditional visual discrimination tasks (Passingham, 1993), in which the subject must select one of two stimuli in order to receive a reward. However, some studies have demonstrated non-conditional discrimination learning impairments following PFC lesions (Gaffan \& Murray, 1990; Parker \& Gaffan, 1998). Human lesion data also support the critical role of PFC on spatial and non-spatial conditional tasks (Petrides, 1985, 1990, 1997; Petrides \& Milner, 1982).

Non-conditional concurrent discrimination learning paradigms have not been widely investigated in frontal lesion patient groups. Equivalent tasks in animals are thought to be performed either using stimulus-reward or stimulus-response associations (e.g. Baxter \& Murray, 2002), and human non-conditional discrimination performance shares a similar ambiguity as regards the exact nature of the representation 
that is acquired. In general, the acquisition of procedural stimulus-response associations is not thought to require PFC recruitment, but is dependent on the striatum (Yin \& Knowlton, 2006). To test this hypothesis, probabilistic learning tasks (Gluck \& Bower, 1988; Knowlton, Squire, \& Gluck, 1994) have been widely used. Patients with Parkinson's disease (PD) and Huntington's disease (HD), who display striatal pathology, show marked impairment on a probabilistic procedural learning task known as the weather prediction task (WPT: Knowlton, Mangels, \& Squire, 1996; Knowlton, Squire, et al. 1996; Shohamy, Myers, Grossman, et al., 2004a; Shohamy, Myers, Onlaor, \& Gluck, 2004b). In the WPT, subjects must predict an outcome (rain or sun) on the basis of four tarot cards, receiving feedback for their response. The outcomes are probabilistically related to the presence or absence of each of the cards (cf. Gluck \& Bower, 1988), with two of the cards being strongly predictive of an outcome and two more weakly. Intact performance was observed in nine patients with frontal lesions (Knowlton, Mangels, et al., 1996), although lesion size and location were not described in detail, and the small group size may have lacked statistical power to detect an impairment. The medial temporal lobe (MTL) may also contribute to WPT performance: a recent study by Hopkins, Myers, Shohamy, Grossman, and Gluck (2004) demonstrated that a group of patients who had suffered bilateral hippocampal damage due to hypoxia were impaired on the task compared to healthy control subjects.

Other evidence has indicated some role for the PFC in procedural learning performance. Electrical (anodal) stimulation of the left PFC (thought to increase neural excitability) using a dc polarisation technique produced a significant improvement on a probabilistic classification learning task (similar to the WPT) in healthy subjects (Kincses, Antal, Nitsche, Bartfai, \& Paulus, 2004). Functional imaging studies have also demonstrated widespread frontal activations during WPT performance (Aron, Gluck, \& Poldrack, 2006; Aron et al., 2004; Fera et al., 2005; Poldrack, Prabhakaran, Seger, \& Gabrieli, 1999; Seger \& Cincotta, 2005), including dorsolateral, inferior, anterior and medial prefrontal regions (Poldrack et al., 1999). The left superior frontal gyrus (BA 8/9) response was associated with behavioural performance (Seger \& Cincotta, 2005). However, it is unclear from functional imaging designs whether these neural responses are actually critical for successful performance. The aim of the present study was to compare learning performance of a large group of frontal patients and non-lesion controls to obtain evidence of the possible role of the prefrontal cortex in stimulus-response or response-reinforcement forms of habit learning thought also to depend on the integrity of the striatum, and sometimes classified as 'procedural' or 'non-declarative' (cf. Knowlton, Mangels, et al., 1996) learning.

We used two learning tasks that have established sensitivity to striatal pathology: the WPT and an eight-pair concurrent discrimination learning task ('Choose': Johnson, Schmitz, Asthana, Gluck, \& Myers, in press; Myers et al., 2002; Shohamy, Myers, Geghman, Sage, \& Gluck, 2006). When seen in the light of fronto-striatal connectivity (Alexander, DeLong, \& Strick, 1986), the similar behavioural profile of patients with frontal and striatal pathologies (e.g. Owen et al., 1992) and the functional relationship between frontal and striatal regions (Pasupathy \& Miller, 2005), Knowlton and colleagues' finding that frontal lesion patients were not impaired on the WPT remains surprising. We recruited a large group of frontal lesion subjects from a patient panel, to address the criticism that earlier studies lacked statistical power and explored learning performance within the frontal lobes in a correlative analysis against the volume of a priori regions of interest (Aron, Fletcher, Bullmore, Sahakian, \& Robbins, 2003). We were particularly interested in patients whose lesions encroached on orbital regions of the prefrontal cortex, as this region is implicated in the processing of stimulus-reward contingencies (Rolls, 2000), and the implication of the ventromedial PFC, which includes the OFC, in nonconditional discrimination learning (Gaffan \& Murray, 1990).

More specifically, anatomically segregated fronto-striatal networks exist which are thought to mediate separate behavioural functions (Alexander et al., 1986). Importantly, the Choose impairment reported by Shohamy et al. (2006) in PD patients was dependent on the medication status of the patients: PD patients tested following taking their dopaminergic medication (i.e. L-dopa) were impaired while unmedicated patients were not. It has been hypothesised that L-dopa medication 'overdoses' fronto-striatal regions with relatively intact dopaminergic input, namely orbital and ventral striatal regions, while improving the functioning of regions with depleted dopamine input (Cools, Barker, Sahakian, \& Robbins, 2001; Swainson et al., 2000). The 'overdose' hypothesis would predict that, given Shohamy's data, Choose performance may be mediated by the orbitofrontal cortex (or the ventral striatum). Preliminary data of Shohamy suggested that WPT performance was not as susceptible to the medication status of PD patients, although performance does seem to depend on PD severity (Knowlton, Mangels, et al., 1996).

The Choose task comprises an initial eight-pair concurrent object discrimination phase (phase 1: 'learn'), in which each discrimination pair differs in one dimension (colour or shape) and shares one relevant dimension (shape or colour, respectively). Subjects learn to pick the correct object of the pair following corrective feedback that is provided after each selection: hence the task shares a similar structure to animal discrimination learning paradigms, although on Choose the subject learns an association with the stimulus and a 'cognitive' reward (a smiling face) rather than a food reward. The task is learned quickly by healthy volunteers, and probably via the use of an explicit rule. One definition of a rule is a categorical decision that is not performed using similarity judgements (Shanks, 1995). Following discrimination acquisition, subjects enter a transfer phase (phase 2: 'transfer') to assess the nature of the discrimination rule that was used in phase 1: subjects perform the same discrimination as in phase 1, but the irrelevant dimension is altered. For example, if in phase 1 the subjects learned to choose a red rather than a blue circle, phase 2 would require the subject to select between red and blue squares. The change between phases is not an intra-dimensional shift where new learning about the currently relevant dimension is required. In Choose, the same stimulus features used to discriminate the pairs on phase 1 apply in phase 2 : only the irrelevant features are replaced. Thus, due to their ability to use 
Stimulus set 1

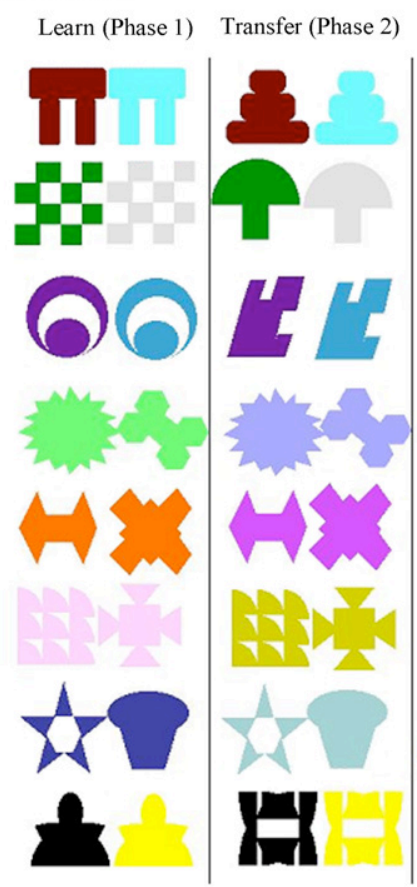

Stimulus set 2

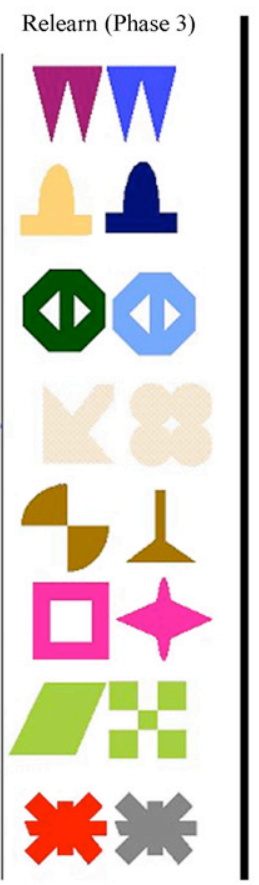

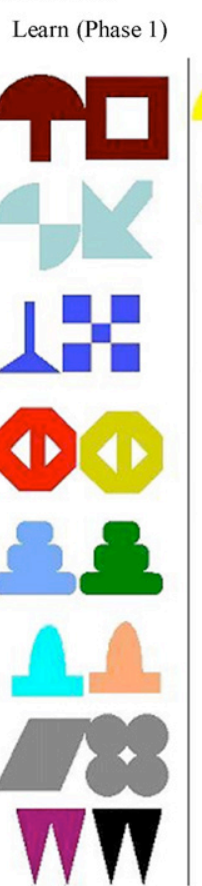

Transfer (Phase 2)

Relearn (Phase 3)
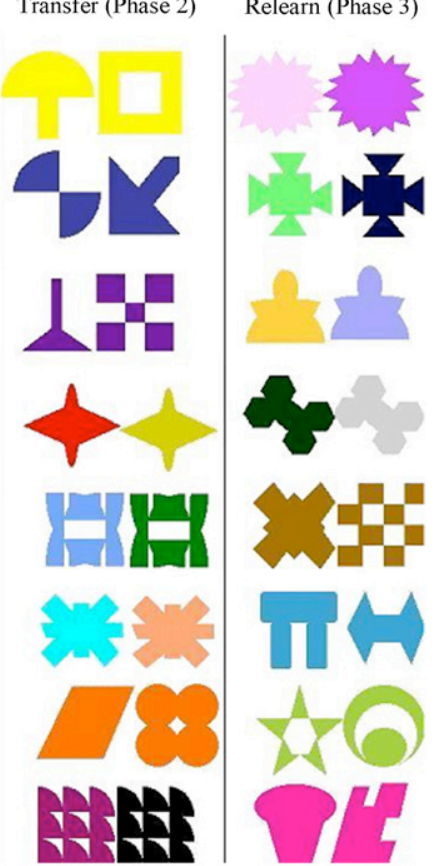

Fig. 1. Stimulus materials employed in Choose. There are two stimulus sets and each subject receives one (selected at random). The diagram shows the stimulus materials for each of the eight pairs for the learning, transfer and relearn phases within each set.

the rule, healthy subjects who pass the learning phase typically make very few errors at transfer as there is no requirement for new learning. Patients with hippocampal atrophy (HA) learn phase 1 quickly but then display transfer impairment (Myers et al., 2002). This suggests that the phase 1 learning in these patients is exemplar-specific rather than rule-based: by changing the irrelevant dimension the similarity between the learned and transfer stimuli is reduced, but the same rule can be used to discriminate the stimuli on both phases. On the basis of this study, HA patients appear to show learning that is specific only to the exemplar used in training and cannot be generalised to novel exemplars that can be categorised with the same rule. Thus the transfer phase of Choose allowed us to test the kinds of representation that the PFC might mediate. If frontal lesion patients' learning was exemplar-specific, as is the case in hippocampal atrophy patients, rather than rule-based, then they should be able to complete phase 1 but be impaired on phase 2. Finally, in phase 3 ('relearn'), subjects are required to learn eight novel pairs of stimuli (Fig. 1).

\section{Methods}

\subsection{Participants}

Patients with lesions of the frontal lobe $(n=36$ : see Table 1$)$ were recruited from the Cambridge Cognitive Neuroscience Research Panel at the MRC Cognition and Brain Sciences Unit. Healthy control subjects $(n=35)$ were recruited from the local community. Controls had no history of psychiatric or neurological disease, and were group-matched to the patient group for age and gender (see Table 1). The national adult reading test (NART, Nelson \& Willison, 1991) was administered to all subjects as a measure of (premorbid) intellectual functioning. All subjects provided informed consent approved by the Addenbrooke's NHS Trust Local Research Ethics Committee. All subjects were paid for their participation. There were some missing data-points due to time constraints and software failure such that in the frontal group, 32 completed WPT and 33 completed Choose, and in the control group, 35 completed WP and 34 completed Choose. All subjects completed at least one task (see Table 1).

\subsection{Lesion characteristics}

Lesion location was confirmed in the frontal group using magnetic resonance imaging (MRI) scanning in a $1.5 \mathrm{~T}$ scanner with $3 \mathrm{D}$ set acquisition, using an SPGR (spin gradient echo) T1-weighted coronal sequence and a T2-weighted axial sequence. Lesions were traced onto each structural scan using MRIcro v1.34 (Rorden \& Brett, 2000) to create a 3D lesion volume, and normalised to the SPM 96 (Statistical Parametric Mapping; Wellcome Department of Cognitive Neurology, London, UK) average T1 structural scan from 152 healthy subjects, using cost function masking (Brett, Leff, Rorden, \& Ashburner, 2001) to exclude the lesion from the calculation of normalisation parameters. Lesion overlaps are displayed in Fig. 2.

Behavioural data were examined in relation to location using two methods. First, given our predictions about the critical role of OFC in learning performance, we subdivided the frontal group into those with damage to this structure and those without damage to this structure by looking for overlap with a region of interest mask encompassing Brodmann area 11 as specified by the MRIcro Brodmann area template. Four patients with one to five voxels of BA11 damage were not included in the OFC group; the subject with the smallest amount of BA11 damage had the equivalent of 119 voxels of damage to that region. Fourteen patients had more than 119 voxels of damage to the OFC, and each of these subjects completed both of the tasks. Two patients within the OFC group had bilateral lesions (i.e. more than 100 voxels damage to each hemisphere of the PFC). We were therefore not able to contrast unilateral and bilateral patients with separate subgroups. Eighteen non-OFC patients completed WPT and 19 non-OFC patients completed Choose.

The two groups were not closely matched for damage to different PFC subregions: the OFC group had suffered more damage to regions proximal to the structure, including the ventrolateral PFC (BA47) and the anterior cingulate cortex (ACC). Although the OFC was central to our hypotheses, we investigated correlations between performance and damage to six other frontal regions of interest, in part to clarify whether an impairment observed within the OFC 
Table

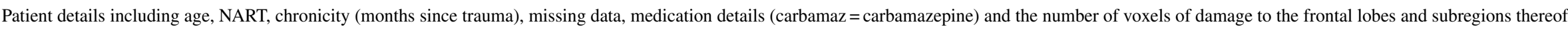
(each voxel has a volume of $2 \mathrm{~mm} \times 2 \mathrm{~mm} \times 2 \mathrm{~mm}$ )

\begin{tabular}{|c|c|c|c|c|c|c|c|c|c|c|c|c|c|c|}
\hline 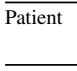 & Aeitology & Age & NART & Chronicity & Completed WPT & Completed Choose & Medication & Frontal & PFC & OFC & Central & Parietal & Temporal & $\begin{array}{l}\begin{array}{l}\text { Basal gan- } \\
\text { glia/thalamus }\end{array} \\
\end{array}$ \\
\hline \multicolumn{15}{|c|}{ Orbitofrontal damage patient group } \\
\hline $1 \mathrm{BL}$ & Tumour & 43 & 121 & 92 & 1 & 1 & Dothiepin & 6,888 & 6,888 & 5062 & 881 & 0 & 0 & 22 \\
\hline $2 \mathrm{R}$ & Tumour & 60 & 116 & 150 & 1 & 1 & Carbamaz & 11,976 & 11,975 & 3025 & 2915 & 0 & 36 & 87 \\
\hline $3 \mathrm{BL}$ & Tumour & 67 & 114 & 180 & 1 & 1 & Atenolol, sinvastatin, lisinopril, bendroflumethazide & 9,736 & 9,736 & 2754 & 485 & 0 & 0 & 0 \\
\hline $4 \mathrm{R}$ & Aneurysm & 61 & 111 & 66 & 1 & 1 & Phenytoin & 7,859 & 7,746 & 2430 & 1860 & 0 & 149 & 97 \\
\hline $5 \mathrm{R}$ & Aneurysm & 53 & 125 & 84 & 1 & 1 & Phenytoin & 6,260 & 6,260 & 2065 & 1920 & 0 & 36 & 247 \\
\hline $6 \mathrm{~L}$ & Aneurysm and infarct & 42 & 121 & 96 & 1 & 1 & & 3,607 & 3,607 & 1636 & 657 & 0 & 1734 & 273 \\
\hline $7 \mathrm{~L}$ & Aneurysm & 52 & 126 & 106 & 1 & 1 & None & 1,321 & 1,321 & 744 & 547 & 0 & 974 & 0 \\
\hline $8 \mathrm{R}$ & Tumour & 51 & 104 & 84 & 1 & 1 & Phenytoin & 4,907 & 4,907 & 632 & 568 & 0 & 0 & 0 \\
\hline $9 \mathrm{~L}$ & Haemorrhage & 51 & 112 & 91 & 1 & 1 & Carbemaz, atenolol, losartan & 483 & 483 & 483 & 73 & 0 & 0 & 0 \\
\hline $10 \mathrm{R}$ & Haemorrhage & 48 & 112 & 30 & 1 & 1 & Epanutin, lamotrigine, citalopram & 664 & 664 & 385 & 6 & 0 & 0 & 0 \\
\hline $11 \mathrm{R}$ & Haemorrhage & 51 & 104 & 54 & 1 & 1 & Bisoprolol, lipitor, lisinopril, lansoprazole, sertraline & 608 & 455 & 142 & 1944 & 0 & 13 & 953 \\
\hline $12 \mathrm{R}$ & Aneurysm & 68 & 121 & 88 & 1 & 1 & None & 2,329 & 2,329 & 140 & 113 & 0 & 0 & 0 \\
\hline $13 \mathrm{R}$ & Aneurysm & 58 & 124 & 84 & 1 & 1 & Carbamaz & 3,951 & 3,750 & 134 & 865 & 0 & 46 & 0 \\
\hline $14 \mathrm{R}$ & Infarct & 58 & 118 & 52 & 1 & 1 & Aspirin, sinvastatin & 3,492 & 2,743 & 119 & 2658 & 0 & 784 & 825 \\
\hline \multicolumn{15}{|c|}{ Non-orbitofrontal damage patient group } \\
\hline $15 \mathrm{R}$ & Tumour and haemorrage & 52 & 119 & 52 & 1 & 1 & Carbamaz & 9,658 & 5,121 & 5 & 8820 & 201 & 0 & 62 \\
\hline $16 \mathrm{~L}$ & Tumour & 61 & 93 & 98 & 1 & 1 & None & 2,065 & 2,065 & 4 & 236 & 0 & 0 & 0 \\
\hline $17 \mathrm{~L}$ & Infarct & 65 & 105 & 48 & 1 & 1 & Aspirin & 6,019 & 5,350 & 1 & 2458 & 0 & 26 & 21 \\
\hline $18 \mathrm{R}$ & Tumour & 30 & 113 & 44 & 1 & 1 & Carbamaz, valproate & 5,891 & 3,655 & 0 & 3337 & 0 & 0 & 0 \\
\hline $19 \mathrm{R}$ & Infarct & 73 & 114 & 82 & 0 & 1 & Warfarin & 308 & 0 & 0 & 389 & 0 & 0 & 0 \\
\hline $20 \mathrm{R}$ & Tumour & 65 & 123 & 11 & 1 & 1 & Benzoflurazide, lipitor & 2,886 & 1,923 & 0 & 1270 & 0 & 0 & 0 \\
\hline $21 \mathrm{R}$ & Tumour & 58 & 111 & 68 & 1 & 1 & Carbamaz, valproate & 376 & 15 & 0 & 421 & 0 & 0 & 0 \\
\hline $22 \mathrm{R}$ & Tumour & 62 & 126 & 26 & 1 & 1 & Lamotrigine, carbamaz, atenolol & 3,283 & 1,412 & 0 & 2508 & 0 & 0 & 0 \\
\hline $23 \mathrm{R}$ & Tumour & 44 & 111 & 72 & 1 & 1 & Lamotrgine & 8,689 & 4,262 & 0 & 6246 & 0 & 0 & 0 \\
\hline $24 \mathrm{R}$ & Tumour & 73 & 120 & 278 & 0 & 1 & Phenytoin & 3,468 & 452 & 0 & 3636 & 0 & 0 & 0 \\
\hline $25 \mathrm{~L}$ & Tumour & 56 & 123 & 84 & 0 & 1 & Fluoxetine & 65 & 65 & 0 & 156 & 0 & 0 & 0 \\
\hline $26 \mathrm{~L}$ & Tumour & 74 & 123 & 40 & 1 & 1 & None & 2,270 & 332 & 0 & 2343 & 0 & 0 & 0 \\
\hline $27 \mathrm{~L}$ & Tumour & 63 & 109 & 84 & 1 & 1 & None & 1,880 & 961 & 0 & 1287 & 0 & 0 & 0 \\
\hline $28 \mathrm{~L}$ & Infarct & 62 & 91 & 106 & 1 & 1 & Aspirin & 820 & 444 & 0 & 394 & 0 & 0 & 0 \\
\hline $29 \mathrm{~L}$ & Infarct & 69 & 124 & 127 & 1 & 1 & Aspirin & 1,882 & 1,882 & 0 & 248 & 0 & 92 & 0 \\
\hline $30 \mathrm{R}$ & Tumour & 47 & 106 & 148 & 1 & 0 & Carbamaz, astorvastatin, ezetimibe, atenolol, ramipril, aspirin & 1,215 & 938 & 0 & 2474 & 0 & 25 & 541 \\
\hline $31 \mathrm{~L}$ & Aneurysm & 62 & 118 & 60 & 1 & 1 & None & 141 & 141 & 0 & 0 & 0 & 1 & 3 \\
\hline $32 \mathrm{R}$ & Infarct & 69 & 123 & 84 & 1 & 1 & Aspirin, simvastatin, bendroflumethazide, amlodipine, perindopril & 315 & 201 & 0 & 122 & 100 & 0 & 0 \\
\hline $33 \mathrm{R}$ & Infarct or haemorrage & 49 & 120 & 144 & 1 & 1 & Phenytoin, lamotrigine & 3,618 & 224 & 0 & 5826 & 101 & 10 & 0 \\
\hline $34 \mathrm{~L}$ & Tumour & 55 & 116 & 90 & 1 & 0 & Valproate, phenytoin & 6,936 & 2,188 & 0 & 8565 & 1669 & 0 & 0 \\
\hline $35 \mathrm{~L}$ & Aneurysm & 60 & 111 & 100 & 1 & 0 & Carbamaz, valproate & 6,866 & 2,776 & 0 & 8262 & 47 & 0 & 50 \\
\hline $36 \mathrm{~L}$ & Tumour & 41 & 123 & 37 & 0 & 1 & Dexamethasone, carbamaz, ranitidine & 4,765 & 3,768 & 0 & 3659 & 246 & 114 & 219 \\
\hline Mean & & 57.03 & 115.30 & & & & & 3,819 & 2,807 & 549 & 2171 & 66 & 112 & 94 \\
\hline S.D. & & 10.14 & 8.65 & & & & & 3,187 & 2,913 & 1143 & 2494 & 280 & 345 & 225 \\
\hline
\end{tabular}




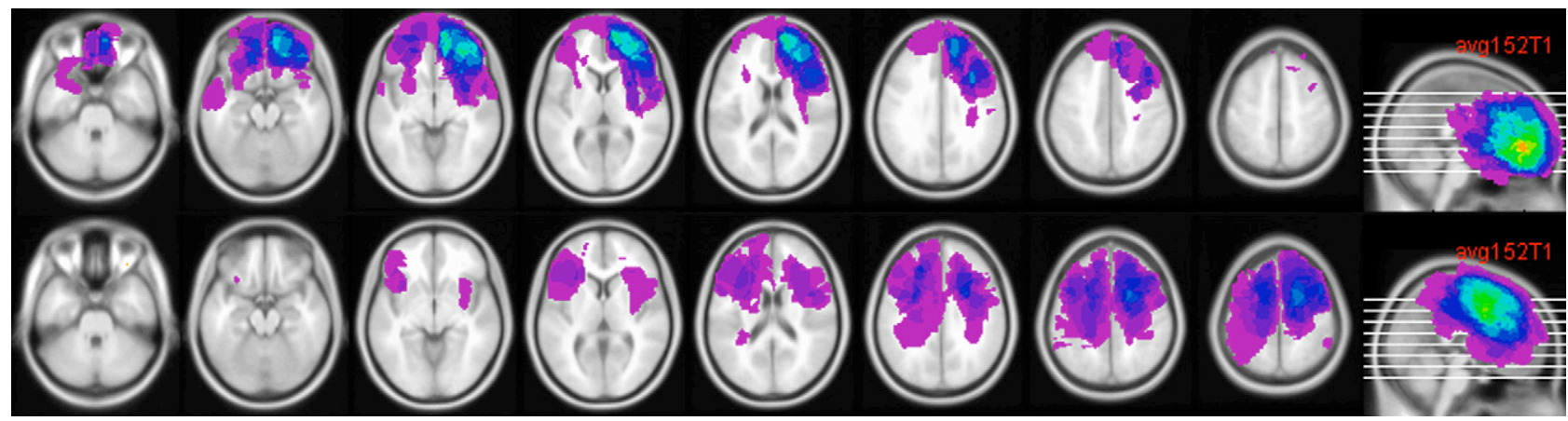

Fig. 2. (Top row) lesion location of OFC lesion patients $(n=14)$; (bottom row) lesion location of non-OFC lesion patients $(n=22)$. Different colours represent different degrees of lesion overlap, coded via colour spectrum (violet represents the least lesion overlap, i.e. one patient, red/orange represents the most lesion overlap). (For interpretation of the references to colour in this figure legend, the reader is referred to the web version of the article.)

group was in fact due to damage to a proximal subregion. The regions of interest were the dorsolateral PFC (DLPFC: BA9 and 46), the inferior frontal cortex (IFC: BA44, 45), the superior frontal cortex (SFC: BA8), the ventrolateral PFC (BA47), the rostral prefrontal cortex (BA10), and the anterior cingulate cortex (ACC: BA 32, 24, 25). We combined the number of voxels of damage to these regions to derive a measure of total prefrontal cortex damage. The combination of total prefrontal cortex damage and total damage to the premotor cortex (BA6) was used to derive a measure of total frontal lobe damage.

In order to rule out the possibility that behavioural changes were associated with non-frontal damage, we calculated the extent of damage to the parietal lobe (BA 5, 7, 39, 40), and the temporal lobe (BA 20, 21, 22, 28, 34-38, 41, 42). We included BA6 in a Central ROI which also included BA 1, 2, 3, 4 and 43. We used the automatic anatomical labelling template of Tzourio-Mazoyer et al. (2002) to estimate subcortical damage, given that there are no Brodmann maps for this region.

\subsection{Behavioural tasks}

Participants completed a behavioural assessment lasting no more than $2 \mathrm{~h}$, administered on a portable PC computer with 10.5 in. touchscreen monitor. Where possible, both tasks were performed during this assessment. In no cases were the two tasks performed on separate occasions.

\subsubsection{Weather prediction task}

The WP task is a 200 trial learning task (see Gluck, Shohamy, \& Myers 2002 for full details). Responses are registered using touch presses on the monitor. On each trial, one to three cards are displayed on the screen, from an array of four possible cards. The four card stimuli each have a unique appearance consisting of an arrangement of circles, squares, triangles or diamonds. There are 14 card combinations ('patterns', see Table 2) in all. On each trial, the subject must make a weather prediction (rainy or sunny) based on the combination of cards presented. After card presentation, the subject has a maximum of $5 \mathrm{~s}$ to respond, with a supplementary cueing signal that appears after $2 \mathrm{~s}$. Feedback is presented $5 \mathrm{~s}$ after card presentation (irrespective of response latency), to inform the subject of their prediction accuracy and the actual weather (i.e. rain or sun). Correct and incorrect predictions increase or decrease a running score (at the side of the screen) by 1 point.

Feedback is determined from a pseudorandom design matrix that is identical for each subject. The four cards are randomly assigned to each condition, for each

Table 2

Associative strengths of each cue

\begin{tabular}{lll}
\hline & $p$ (sun $\mid$ cue present) & $p$ (rain $\mid$ cue present) \\
\hline Cue 4 & 0.756 & 0.244 \\
Cue 3 & 0.575 & 0.425 \\
Cue 2 & 0.425 & 0.575 \\
Cue 1 & 0.244 & 0.756 \\
\hline
\end{tabular}

subject. The design matrix is organised such that one cue is strongly associated with rain (76\%) rather than sun (24\%), one cue is strongly associated with sun (76\%) rather than rain (24\%), and the other two cues are more weakly paired with those respective outcomes (see Table 1). Data are analysed in terms of a percent correct score: choosing the outcome which is most likely to give positive feedback (over the course of the entire experiment) is a correct answer. Trials where two cards with equal associative strength are present for opposite outcomes (e.g. patterns $\mathrm{F}$ and I, see Table 2) are excluded from the analysis (Table 3).

\subsubsection{Choose}

Choose is a concurrent discrimination learning task using touchscreen control (see Myers et al., 2002 for more details). The task stimuli consist of eight pairs of objects, of which four pairs have the same shape and can be differentiated by colour, and the other four have the same colour and can be differentiated by shape. One member of each pair is randomly assigned to be correct. One each trial, the subject is asked to select one of the two objects; the chose object is raised on the screen and, if the response was correct a smiling face appears beneath. Trials with the eight discrimination pairs are intermixed in the acquisition phase (phase 1). Once phase 1 has been learned to a criterion of 16 correct responses in a row (or to a maximum of 96 trials), the dimension common to each stimulus is changed. For example, if the discrimination is performed on the basis of colour, then the shape of the object would change during the transfer stage, and vice versa. The same learning criterion of 16 consecutive correct responses (within a maximum of 48 trials) was set for phase 2 . If the subject did not pass either phase 1 or 2 the subject would move on to the following phase anyway (i.e. phase 2 or 3 , respectively). Phase 3, a relearning phase, requires the subject to learn the correct response when presented with eight new pairs

Table 3

Associative strengths of each pattern

\begin{tabular}{lllllll}
\hline Pattern & Cue 1 & Cue2 & Cue 3 & Cue 4 & $p$ (pattern) & $p$ (rain $\mid$ pattern) \\
\hline A & 0 & 0 & 0 & 1 & 0.14 & 0.143 \\
B & 0 & 0 & 1 & 0 & 0.08 & 0.375 \\
C & 0 & 0 & 1 & 1 & 0.09 & 0.111 \\
D & 0 & 1 & 0 & 0 & 0.08 & 0.625 \\
E & 0 & 1 & 0 & 1 & 0.06 & 0.167 \\
F & 0 & 1 & 1 & 0 & 0.06 & 0.500 \\
G & 0 & 1 & 1 & 1 & 0.04 & 0.250 \\
H & 1 & 0 & 0 & 0 & 0.14 & 0.857 \\
I & 1 & 0 & 0 & 1 & 0.06 & 0.500 \\
J & 1 & 0 & 1 & 0 & 0.06 & 0.833 \\
K & 1 & 0 & 1 & 1 & 0.03 & 0.333 \\
L & 1 & 1 & 0 & 0 & 0.09 & 0.889 \\
M & 1 & 1 & 0 & 1 & 0.03 & 0.667 \\
N & 1 & 1 & 1 & 0 & 0.04 & 0.750
\end{tabular}

0 represents card absence and 1 , the card presence. Patterns 1111 and 0000 were not used. 
Table 4

Demographic information and WPT and Choose performance for patients and controls

\begin{tabular}{lcc}
\hline & Patient & Control \\
\hline Age & $57.03(10.14)$ & $55.37(11.54)$ \\
NART & $115.30(8.65)$ & $116.65(5.17)$ \\
WPT quarter 1 (trials 1-50) & $0.61(0.15)$ & $0.70(0.16)$ \\
WPT quarter 2 (trials 51-100) & $0.67(0.15)$ & $0.71(0.16)$ \\
WPT quarter 3 (trials 101-150) & $0.67(0.16)$ & $0.75(0.15)$ \\
WPT quarter 4 (trials 151-200) & $0.74(0.15)$ & $0.75(0.16)$ \\
Choose learn errors & $18.27(14.02)$ & $13.26(13.96)$ \\
Choose relearn errors & $13.55(14.00)$ & $10.53(12.97)$ \\
\hline
\end{tabular}

The patient $(n=32)$ and controls $(n=35)$ completing the WPT could not be distinguished by age $(t=0.458$, d.f. $=65, p=0.649)$ or NART $(t=-1.073$, d.f. $=48.74, p=0.289)$, neither could the patients $(n=33)$ and controls $(n=34)$ completing Choose be distinguished by age $(t(65)=0.526, p=0.643)$ or NART $(t(51.70)=-0.466, p=0.604)$.

of stimuli to assess. Learning criterion for phase 3 is identical to phase 1 . Thus there are 24 pairs of stimuli that constitute the stimulus material for all three phases. There were two sets of 24 pairs, and each subject is randomly assigned to receive one. Measures extracted from the task is a binary score for each phase reflecting whether the subject reached the criterion of 16 correct in a row, and the errors accrued during learning of that stage.

\subsection{Statistical analysis}

Due to some patients failing to complete either task, one-way analysis of variance (ANOVA) for age and NART IQ were conducted separately for each task to ensure adequate matching across groups. Behavioural performance was analysed using ANOVA with group as a two or three-level between-subjects variable (controls, patients; controls, OFC, non-OFC). The Greenhouse-Geisser correction was applied when the homogeneity of variances was violated. For the WPT, percentage correct data were analysed for each Quarter, or block of 50 trials (four levels) as a within subjects factor, and an analysis of 10-trial blocks over the first 50 trials (five levels) as a further within subjects factor. For Choose, the number of errors were assessed in the acquisition and relearn phases was compared across groups (following Myers et al., 2002; Shohamy et al., 2006). Performance at the transfer phase was compared across groups for subjects who had passed the initial acquisition phase. Error scores for Choose were $\log$ transformed as raw scores were highly skewed. Untransformed values are presented in tables and figures. In addition, following Shohamy et al. (2006) and Myers et al. (2002), we also analyzed whether the subject passed criterion or not on the learn and relearn phases: this was done with a Fisher's exact test on each phase separately.

To examine the effect of lesion size, Pearson correlation coefficients were calculated for the associations between learning performance (collapsed across the within subjects variables) and MRI volumes (total lesion size or region of interest damage). All statistics were thresholded at $p<0.05$ (two-tailed).

\section{Results}

\subsection{Choose}

See Tables 4 and 5.

\subsubsection{Effect of damage to orbitofrontal cortex (Fig. 3)}

We performed two Fisher's exact tests contrasting pass and fail rates for each of the three groups (OFC, non-OFC, controls) on the learn phase and relearn phase. The Fisher's exact statistic was significant for each test (learn phase $=8.549, p=0.011$; relearn phase $=15.708, p<0.001$ ). Repeated measures ANOVA of error scores on the learn and relearn phases also revealed a
Table 5

Pass and fail rates for patients and controls on the learn and relearn phase of Choose

\begin{tabular}{lrllllll}
\hline & \multicolumn{2}{l}{ Learn } & & & & \multicolumn{2}{l}{ Relearn } \\
\cline { 2 - 3 } \cline { 8 - 9 } & Fail & Pass & Total & & Fail & Pass & Total \\
\hline Patients & 14 & 19 & 33 & & 10 & 23 & 33 \\
Controls & 5 & 29 & 34 & & 5 & 29 & 34 \\
Total & 19 & 48 & 67 & & 15 & 52 & 67 \\
\hline
\end{tabular}

significant main effect of group $(F(2,64)=5.411, p=0.007)$, of phase $(F(1,64)=6.551, p=0.013)$, and a group by phase interaction $(F(2,64)=3.456, p=0.038)$. Tukey's HSD post hoc tests showed that subjects with OFC damage were impaired compared to both controls $(p=0.008)$ and non-OFC frontals $(p=0.016)$. Non-OFC frontals were not significantly different from controls $(p=0.925)$. In a simple effects analysis of the group by phase interaction, both controls $(t(33)=2.528, p=0.016)$ and the non-OFC patients $(t(18)=2.446, p=0.025)$ showed a significant improvement on the relearn phase compared to the learn phase, whereas the OFC patients performed equivalently on both phases $(t(13)=-0.829, p=0.422)$. Univariate ANOVA revealed that the three groups could not be distinguished on the transfer phase, if data from the only subjects who passed the learn phase were included (OFC: $n=6$, non-OFC: $n=13$, control: $n=29$; $F(2,47)=0.546, p=0.583)$. Overall, all groups made very few errors on this phase, provided they had passed the learning phase (mean transfer errors: OFC patients 2.17 ; non-OFC patients 1.46; controls 1.38).

Student's $t$-tests were used to clarify the nature of the impairment: OFC patients were impaired on both the learn and relearn phase compared to controls (learn: $t(46)=2.059, p=0.045$; relearn: $t(46)=3.364, p=0.002)$, but compared to non-OFC patients the impairment reached significance only on the relearn phase (learn: $t(31)=1.191, p=0.243$; relearn: $t(31)=4.817$, $p<0.001)$. Non-OFC patients were not significantly different from controls on either phase $(t(51)<1$ in both cases).

Frontal patients with OFC damage had a significantly greater volume of damage to the PFC than those without damage to that

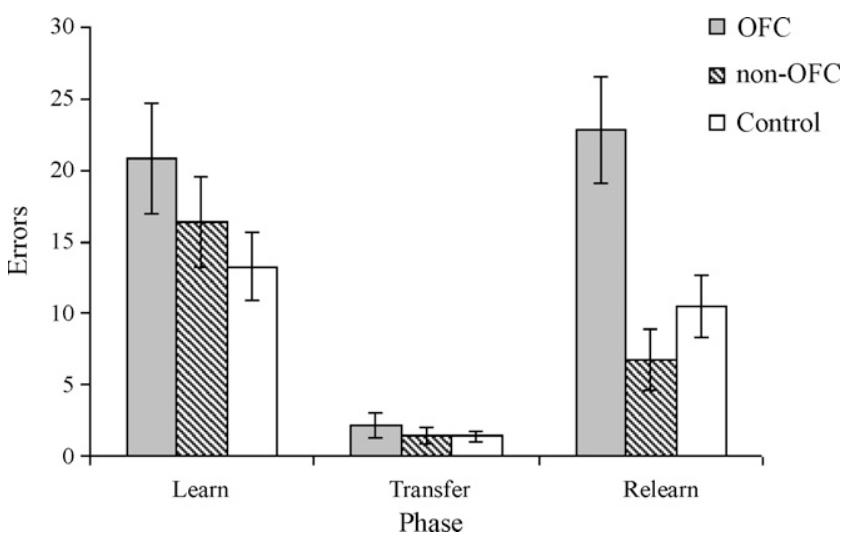

Fig. 3. Raw error scores for OFC lesion patients (grey), non-OFC lesion patients (hatched) and controls (white) on the learning and relearning phases of Choose. Transfer data only includes subjects who passed the learn phase of Choose. 
region (OFC lesion: mean 4490.29 voxels, S.D. 3608.45; nonOFC lesion: mean 1698.58 voxels, S.D. $1837.85 ; t(31)=2.652$, $p=0.016$ ). We compared the two frontal groups (OFC, non-OFC lesion patients) with a repeated measure ANOVA and included total volume of PFC damage as a covariate. Both the group effect $(F(1,30)=6.718, p=0.015)$, and the group by phase interaction remained intact $(F(1,30)=7.713, p=0.009)$. To further control for differences in lesion volume, we excluded the five OFC patients with the largest lesions, such that the total PFC lesion volumes of the OFC and non-OFC groups were now reasonably matched (OFC lesion: mean 2251.00 voxels, S.D. 1624.93; non-OFC lesion: mean 1698.58 voxels, S.D. 1837.85; $t(26)<1)$. The group and group by phase interaction effects previously observed following a repeated measures ANOVA of the learn and relearn phases remained significant: there was a significant main effect of group $(F(2,59)=4.311, p=0.018)$, of phase $(F(1,59)=3.891, p=0.053)$, and a group by phase interaction $(F(2,59)=3.169, p=0.049)$. Tukey's HSD post hoc tests revealed that subjects with OFC damage were impaired compared to both controls $(p=0.019)$ and non-OFC frontals $(p=0.027)$. Non-OFC frontals were not significantly different from controls $(p=0.995)$.

\subsubsection{Effect of damage to prefrontal subregions}

We performed a correlational analysis to investigate the possible contribution of other prefrontal subregions (superior frontal gyrus (BA8); ventrolateral PFC (BA47); inferior frontal gyrus (BA44/45); dorsolateral PFC (BA46/9); rostral PFC (BA10); anterior cingulate cortex (ACC: BA32/24/25)), and the effect of total PFC lesion volume on total error scores. None of the correlations of total Choose error scores with lesion volume to each subregion reached significance (highest $r$ value $=0.287$ ), neither did the effect of total PFC ( $r=0.298, n=33, p=0.092)$ or frontal lobe damage $(r=0.270, n=33, p=0.128)$.

\subsubsection{Effect of damage to non-frontal regions}

An analysis was performed to confirm the lack of effect of damage to non-frontal areas (parietal, temporal, basal ganglia/thalamus) correlations between performance and damage to the regions were performed. No effect of damage to these regions was observed (Appendix A, Table A2). Also, we compared patients with and without damage to the temporal lobes, parietal lobes and subcortical areas (basal ganglia and thalamic regions); these analyses revealed no significant differences among patients with or without damage to these areas (maximum $r=0.31$, maximum $t=1.784$ ).

In summary, OFC lesions were associated with a deficit in Choose performance, whereas non-OFC frontal lesion patients could not be distinguished from controls. This deficit could not be explained by differences in lesion volume between the two groups, or by damage to non-frontal regions.

\subsubsection{Error analysis}

We categorised errors in terms of whether they had followed reward omission or not. Errors that followed an incorrect response to a given stimulus pair were classified as lose-stay errors, while errors that followed a correct response to a given pair were win-shift errors. A repeated measures ANOVA model including group, phase (learn, relearn) and error type revealed a significant main effect of error type $(F(2,64)=8.351$, $p=0.005)$, as subjects made more lose-stay errors than win-shift errors. The main effect of group remained significant $(F(2$, $64)=5.407, p=0.007)$, as did the group by phase interaction $(F(2,64)=4.387, p=0.016)$. The further interaction terms by error type were not significant (type by group $(F(2,64)=1.750$, $p=0.182)$; type by phase by group $(F(2,64)=2.295, p=0.109)$.

\subsection{Weather prediction}

\subsubsection{Effect of damage to the OFC (Fig. 4)}

A further analysis distinguished frontal patients with and without OFC damage. Using data from all 200 trials, a repeated measures ANOVA of task quarter revealed no significant main effect of group $(F(2,64)=1.668, p=0.197)$ or group by quarter interaction $(F(5.187,165.970)=1.562, p=0.171)$. On the first 50 trials there was a main effect of group $(F(2$, $64)=3.367, p=0.041$ ) but no group by 10 trial block interaction $(F(6.638,212.423)=0.494, p=0.829)$. Tukey's HSD post hoc tests showed OFC patients and controls differed significantly $(p=0.038)$. However, contrasts between non-OFC patients and controls $(p=0.524)$, and OFC and non-OFC patients $(p=0.347)$ were not significantly different in either case. There was a time limit for responding on the weather prediction task. In general, the overall number of timeouts were small (mean timeouts over 200 trials: OFC patients: 2.09 ; non-OFC patients: 1.31 ; controls: 1.19 ) and could thus not drive the effects we observed.

\subsubsection{Effect of damage to prefrontal regions}

We performed a correlational analysis to investigate the possible contribution of other prefrontal subregions (superior frontal gyrus (BA8); ventrolateral PFC (BA47); inferior frontal gyrus (BA44/45); dorsolateral PFC (BA46/9); rostral PFC (BA10); anterior cingulate cortex (ACC: BA32/24/25)), and the effect of total PFC lesion volume on total error score. None of the correlations of percent correct with lesion volume to each subregion reached significance (largest $r$ value $=-0.250$ ), neither did the effect of total PFC ( $r=-0.179, n=32, p=0.328)$ or frontal lobe damage $(r=-0.213, n=32, p=0.242)$.

\subsubsection{Effect of damage to non-frontal regions}

We assessed whether these behavioural differences were associated with non-frontal damage. Comparison of patients with and without damage to the temporal lobes, for parietal lobes and subcortical areas (basal ganglia and thalamic regions) revealed no significant differences (maximum $t=1.28$ ), and there were no significant correlations between performance and the volume of damage to these regions (maximum $r=-0.21$ : see Appendix A, Table A1).

In summary, frontal lesions patients showed a mild impairment on the first 50 trials of the WPT, but not overall. OFC patients performed significantly worse than controls on the first 50 trials, but not significantly worse than non-OFC lesion patients. Although there was not a selective deficit on the WPT in OFC patients, it was not evident that damage to 

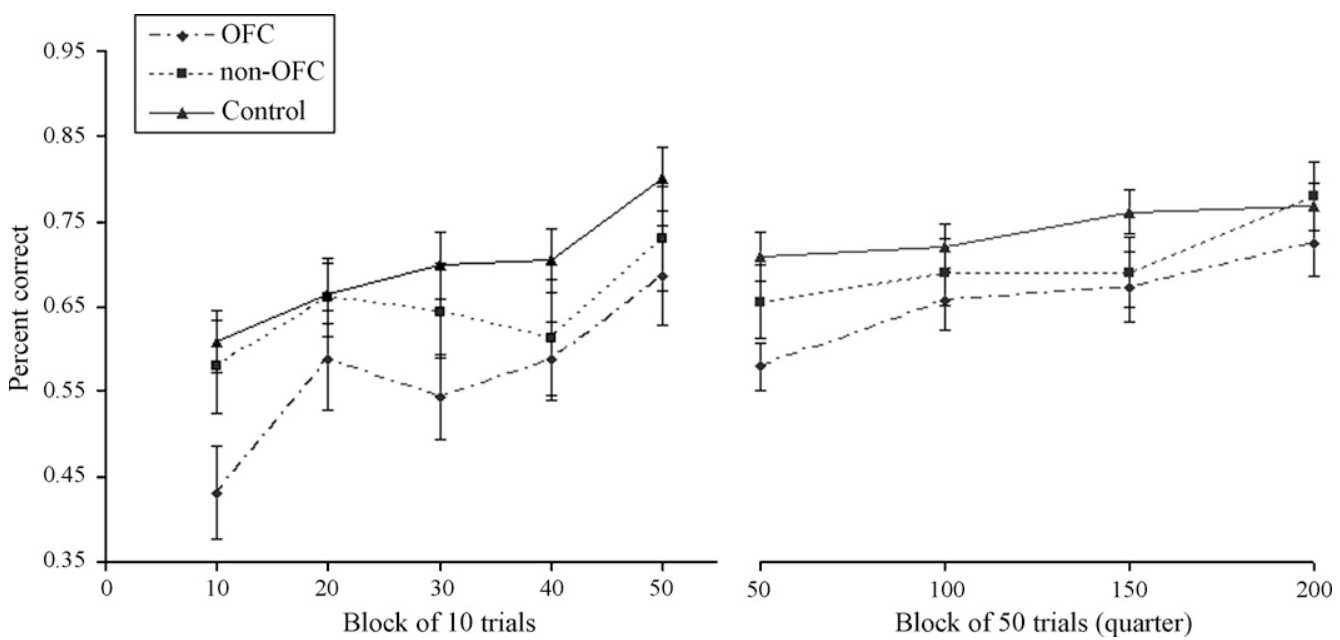

Fig. 4. Performance of OFC (dot-dash), non-OFC (dotted line) and controls (complete line) on the first quarter (50 trials, left) and the whole 200 trials (right) of the weather prediction task.

another PFC subregion resulted in a clearer impairment. Damage to non-frontal regions had no detectable effect on WPT performance.

\subsection{Summary}

In summary, patients with OFC damage showed clear learning impairments on Choose, compared to controls and non-OFC frontal lesion patients. These patients also showed a mild impairment on the first 50 trials of the WPT, but performed equivalently to controls over the whole 200 trials. However, there was no evidence for a selective deficit in these patients compared to other patients with frontal lesions on the first 50 trials.

\section{Discussion}

In this study, we examined the learning performance of frontal lesion patients and controls on two learning tasks with established sensitivity to striatal pathology, the Weather Prediction Task (WPT, Knowlton et al., 1994) and the eight-pair concurrent visual object discrimination learning task, 'Choose' (Myers et al., 2002). We present evidence that the integrity of the orbitofrontal region of the frontal cortex plays a role in normal learning performance on the Choose task. If the frontal group was divided depending on whether the patient had suffered damage to the OFC or not, a highly significant effect of group emerged, with the OFC group being impaired on Choose compared to both controls and non-OFC frontals.

Deficits on discrimination acquisition on Choose in patients with striatal pathology (i.e. Parkinson's disease, PD) were shown previously to be dependent on dopamine medication: medicated but not unmedicated PD patients were impaired on the task (Myers et al., 2002; Shohamy et al., 2006). It has been suggested that dopaminergic medication may 'overdose' cognitive functions dependent upon ventral striatal-orbitofrontal circuitry (Cools et al., 2001; Cools, Barker, Sahakian, \& Robbins, 2003; Cools, Lewis, Clark, Barker, \& Robbins, 2007; Swainson et al.,
2000), which are relatively intact in the early stages of PD (Kish, Shannak, \& Hornykiewicz, 1988). Our finding that damage to the OFC leads to a similar deficit to L-dopa medication in PD is consistent with this view. On the other hand, the transfer phase of the Choose task was shown previously to depend on medial temporal lobe integrity (Myers et al., 2002); neither the OFC nor non-OFC frontal subgroups displayed deficits on this stage of the task.

In contrast with the data of Knowlton, Mangels, et al. (1996), we observed that frontal lesion patients can show WPT deficits on the first 50 trials: specifically, OFC but not non-OFC patients were impaired at this stage. However, OFC and non-OFC patients did not differ significantly (so it was not possible to ascribe a particular role for the OFC in WPT performance). It is possible that Knowlton et al. failed to see an impairment in their frontal lesion patients due to a lack of power: we estimated that the frontal vs. control effect size in that study was 0.66 , and the power to detect a difference was 0.32 . There were also likely to be differences in lesion size or location from the patients tested in the present study compared to the group tested by Knowlton. These authors did not split their frontal group in terms of different PFC subregions, and the effect size that they report is somewhere in between the effect size of our nonOFC group (0.31) and our OFC group (0.84). On the basis of this study, we predict that detecting a significant difference in future studies comparing controls with frontal lesion patients using the WPT may be difficult: the power to detect a significant difference with a non-OFC frontal group we estimate is 0.17 , and an OFC lesion group is 0.74 . In our data, there were no significant differences in WPT performance between the OFC and non-OFC lesion groups, and we also saw no significant correlations between the volume of damage to frontal subregions and WPT performance. As with PD, it may be that WPT performance is dependent on the severity of the patient's impairment. WPT impairment may become more likely if a number of processes mediated by the PFC are compromised. Our inability to observe a correlation with volume of PFC lesion 
may have resulted from the inherent variability of the WPT measure.

It is worth noting that control performance of the WPT has a large variance, with a number of controls performing around chance throughout the experiment, which makes detecting small group differences difficult. The only procedural difference between our implementation of the WPT and the protocol used by Knowlton was that we used a pseudorandom sequence, whereas Knowlton et al. used a fully random sequence. We did this in attempt to enhance the sensitivity of the task, by reducing intra-group variance in performance. The effect that we detected in the first 50 trials may have arisen as a result of this enhanced sensitivity.

The principal limitation of the study was the relative lack of selectivity of the patients' lesions. The OFC patients had larger overall lesion volume than the non-OFC group, and had also sustained more damage to adjacent sectors of the PFC including the ventrolateral region (BA47) and the anterior cingulate cortex. As such, it is uncertain whether OFC damage alone is sufficient to produce discrimination learning deficits, as to display task deficits may require a combination of damage to the OFC and another prefrontal region.

Previous studies of non-conditional object learning in monkeys have emphasised the importance of bilateral lesions of the PFC for disrupting this behaviour (Gaffan \& Murray, 1990; Parker \& Gaffan, 1998). The majority of our patients - all but two - were unilateral lesion patients. We did not have a strong hypothesis about different roles for the two hemispheres in mediating performance of the two tasks we employed, though there is neuroimaging evidence that the two hemispheres can have dissociable roles in learning performance (Fletcher, Buchel, Josephs, Friston, \& Dolan, 1999). One critical aspect of the bilateral lesion is that the function of the region is compromised in its entirety, rendering arguments about redundant processing within a region irrelevant. However, all the lesion patients in this study had considerable intact tissue in the OFC (even those with OFC lesions): our data supports the notion that Choose is a sensitive measure of OFC integrity (perhaps in combination with damage to other PFC subregions) and that reductions in OFC processing capacity due to lesions can be registered in Choose performance. Future research may usefully explore the role of lesion laterality in learning performance.

A number of patients had damage to non-frontal regions (as assessed by standardised neurological templates), including the parietal lobes, temporal lobes and subcortical regions, including the basal ganglia and thalamus. There was no evidence in the present data set that non-frontal damage affected performance on either task. Clearly this does not contradict the body of evidence demonstrating that extensive lesions to the striatum or temporal lobe, in particular, compromise learning performance (Hopkins et al., 2004; Knowlton, Mangels, et al., 1996; Knowlton, Squire, et al. 1996; Meeter, Myers, Shohamy, Hopkins, \& Gluck, 2006; Myers et al., 2002). We simply wished to rule out the possibility that the effects observed were driven by differences between the lesion groups in the degree of damage to non-frontal regions. Our data are not easily comparable with these previous studies: for example, PD and HD are neurodegenerative diseases which may alter basal ganglia function in a different manner to a lesion (see, for example, Frank (2005) for one account of how PD pathology might affect WPT performance). Moreover, the lesions described to the non-frontal regions are not selective and variable in extent and volume (often being small), and therefore do not offer decisive evidence as to the contribution of these regions to WPT or Choose performance. Studies along the lines of Ell, Marchant, and Ivry (2006) would be more promising in this regard.

In a previous study of non-conditional learning in human frontal lesion patients, Swainson et al. (2006) found that patients and controls performed equivalently on an eight-pair concurrent discrimination learning task. The authors did not investigate the contribution of different PFC subregions to this behaviour or use a criterion as a dependent measure, and thus our data are consistent with their findings. However, Choose and the discrimination learning task employed by Swainson et al. have shown differential sensitivity in other patient groups: Shohamy et al. found Choose acquisition performance to be impaired in medicated but not unmedicated PD, whereas Swainson et al. did not observe a differential effect of medication on acquisition performance in PD. Although different aetiologies or lesion locations within the temporal lobe may well explain these results, it is worth noting that Swainson et al. found temporal lobe lesion patients to be impaired on their task, whereas Myers et al. found hippocampal atrophy patients to be intact on Choose acquisition (Myers et al., 2002). The visual properties of the stimuli, which vary strikingly between the tasks employed by Swainson and Myers/Shohamy, could be the critical variable determining differential sensitivity. Indeed, this variable has been investigated as part of an ongoing study of medial temporal lobe function, specifically with regard to the role of the perirhinal cortex in discriminating stimuli with overlapping features (Bussey, Saksida, \& Murray, 2002).

The impaired performance of OFC patients on Choose is surprising in the context of data from experimental animals and human lesion patients demonstrating intact discrimination acquisition performance (i.e. selecting $\mathrm{A}$ of an $\mathrm{AB}$ pair when $\mathrm{A}$ is rewarded and B is not rewarded) contrasting with impaired reversal performance (i.e. where $\mathrm{A}$ is now not rewarded and $\mathrm{B}$ is rewarded e.g. (Butter, 1969; Chudasama \& Robbins, 2003; Dias, Robbins, \& Roberts, 1996; Hornak et al., 2004; Iversen \& Mishkin, 1970). A possible account for the discrepancy is that reversal is normally a faster process than initial acquisition because the task relevant information becomes evident to the subject following acquisition (Kruschke, 1996). However, Choose is acquired very quickly by healthy control subjects. The ease of performance of Choose may well be a function of the fact that the stimuli used in Choose are relatively easy to verbalise and perhaps therefore to encode: the shapes and colours of the stimuli are fairly familiar. It is possible that, on tasks where the stimulus materials are sufficiently easy to encode, fast acquisition is mediated by the OFC, and hence impaired following OFC lesions in the same way as is reversal learning. On tasks which are learned more slowly than Choose, such as that employed by Swainson et al., we would not expect a system facilitating fast learning to have a critical role in behaviour, and therefore we 
would not expect OFC damage to have a detectable impact on behaviour.

Subjects were required to learn two sets of eight-pair object discrimination problems: OFC patients were more clearly impaired on the second of the two pairs, although these patients were mildly impaired on the first phase compared to controls. One account of the pattern of data, given that the OFC group did not improve performance on the second of the two sets, whereas the other two groups did, is that the OFC lesion patients did not demonstrate the development of a 'learning set' (as observed by (Harlow, 1949) in monkeys). OFC patients, unlike non-OFC patients or controls, were not able to improve performance on the second set of stimuli compared to the first. There are a variety of mechanisms that may account for the learning set effect (Kehoe, 1988): for example, learning set may facilitate learning by focussing the subject's attention on relevant stimulus features (Mackintosh, 1975), or via encoding differences dependent on prospective memory (Murray \& Gaffan, 2006). Our pattern of results (main effect of lesion type and lesion type by phase interaction) is compatible with the suggestion that the OFC might be involved in one or more of these processes: due to the fact that Choose can be acquired rapidly, it is likely that such processes contribute to learning phase (phase 1) performance, but are more evident on the relearn phase, where learning set is facilitating performance in the non-OFC patient and control groups. There is other evidence that the learning set effect is dependent on the PFC (Browning, Easton, \& Gaffan, 2007; Harlow, Davis, Settlage, \& Meyer, 1952), and some evidence implicating the OFC (Yokoyama, Tsukada, Watanabe, \& Onoe, 2005). In a recent review, Wallis (2007) describes a small body of evidence that the OFC contributes to discrimination learning performance in experimental animals in terms of the region implementing the strategic control of learning performance. Learning set may include mnemonic strategies: thus this position is compatible with our view.

Given that the task is performed via the association between a stimulus and affective outcome (smiling face, smiling face omission), our results are compatible with electrophysiological recordings from OFC neurons which suggest that neurons in this region are sensitive to the value of expected rewards (Roesch \& Olson, 2004; Schoenbaum, Setlow, \& Ramus, 2003; Tremblay \& Schultz, 1999; Wallis \& Miller, 2003). Particularly pertinent is the work of Wallis and Miller (2003), in which OFC neurons were found to encode the identity of individual visual stimuli predictive of reward, and also those which encoded both the stimulus identity and its associated reward value.

As the task is learned so rapidly by control subjects, it is possible that explicit recognition of the discriminanda plays a role in their association with reward or reward omission, and that these processes are subserved by the OFC. The visual recognition functions of the OFC are often not emphasised but are nonetheless supported by the published literature (Bar et al., 2006; Frey \& Petrides, 2000; Meunier, Bachevalier, \& Mishkin, 1997). Extensive connections between the temporal cortex and the OFC (e.g. Barbas, 1993; Webster, Bachevalier, \& Ungerleider, 1994) may mediate such a function.

In conclusion, OFC lesion patients were impaired on eightpair concurrent discrimination learning compared to control and non-OFC frontal lesion patients. Learning performance of the latter two groups could not be distinguished on this task. Patients with damage to the OFC also showed additional subtle impairments on the weather prediction task over the first 50 trials compared to controls (but not non-OFC patients), which contrast however with the severe deficits observed previously in patients with basal ganglia damage. These results provide evidence that frontal lesion patients display hetereogeneous learning performance, but that the orbitofrontal subregions may contribute to normal discrimination learning in human subjects.

\section{Acknowledgements}

This work was funded by a Wellcome Trust Programme Grant to T.W.R., B.J.S., B.J. Everitt and A.C. Roberts, and completed within the University of Cambridge Behavioural and Clinical Neuroscience Institute, supported by a consortium award from the Medical Research Council (UK) and the Wellcome Trust. Many thanks to the Cambridge Cognitive Neuroscience Research Panel, Dr. N. Antoun, Dr. F. Manes and Dr. P.C. Fletcher for radiological tracing and assistance with structural MRI analyses. We are also grateful to the members and staff of the Cambridge Cognitive Neuroscience Research Panel (CCNRP). HWC is supported by a 3-year MRC studentship.

\section{Appendix A}

See Tables A1 and A2.

Table A1

Pearson's correlation coefficients and t statistics relating WPT performance to damage to different brain regions

\begin{tabular}{|c|c|c|c|c|c|}
\hline Region & Quarter 1 & Quarter 2 & Quarter 3 & Quarter 4 & Quarter $1 t$-stat ( $p$ value) \\
\hline Total PFC & -0.18 & -0.04 & 0.03 & 0.03 & \\
\hline Central & -0.21 & -0.18 & -0.13 & -0.13 & \\
\hline Parietal & -0.17 & -0.12 & -0.11 & -0.20 & $<1$ \\
\hline Temporal & -0.03 & -0.19 & 0.04 & 0.00 & $-1.28(0.211)$ \\
\hline $\mathrm{ACC}$ & -0.14 & -0.08 & -0.11 & -0.16 & $-1.21(0.237)$ \\
\hline Basal ganglia/thalamus & 0.05 & 0.17 & 0.20 & 0.17 & $<1$ \\
\hline
\end{tabular}

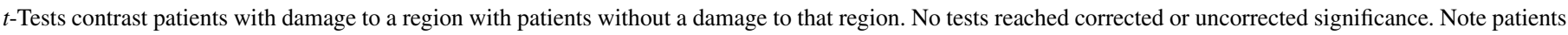
with central lesions are numerically superior to patients without lesions to that region. $N=32$ in all cases. 
Table A2

Pearson's correlation coefficients and t statistics relating Choose performance to damage to different brain regions

\begin{tabular}{lrrc}
\hline Region & Learn & Relearn & Total errors: $t$-test \\
\hline Total PFC & 0.27 & 0.27 & \\
Central & 0.17 & -0.09 & \\
Parietal & 0.13 & -0.09 & $<1$ \\
Temporal & -0.06 & 0.15 & $1.784(0.084)$ \\
ACC & 0.31 & -0.12 & $1.192(0.242)$ \\
Basal ganglia/thalamus & -0.02 & 0.14 & $<1$
\end{tabular}

$\overline{t \text {-Tests contrast patients with damage to a region with patients without a damage }}$ to that region. No correlations reached corrected significance. $N=33$ in all cases.

\section{References}

Alexander, G. E., DeLong, M. R., \& Strick, P. L. (1986). Parallel organization of functionally segregated circuits linking basal ganglia and cortex. Annual Review of Neuroscience, 9, 357-381.

Aron, A. R., Fletcher, P. C., Bullmore, E. T., Sahakian, B. J., \& Robbins, T. W. (2003). Stop-signal inhibition disrupted by damage to right inferior frontal gyrus in humans. Nature Neuroscience, 6(2), 115-116.

Aron, A. R., Shohamy, D., Clark, J., Myers, C., Gluck, M. A., \& Poldrack, R. A. (2004). Human midbrain sensitivity to cognitive feedback and uncertainty during classification learning. Journal of Neurophysiology, 92(2), $1144-1152$.

Aron, A. R., Gluck, M. A., \& Poldrack, R. A. (2006). Long-term test-retest reliability of functional MRI in a classification learning task. Neuroimage, 29(3), 1000-1006.

Bar, M., Kassam, K. S., Ghuman, A. S., Boshyan, J., Schmid, A. M., Dale, A. M., et al. (2006). Top-down facilitation of visual recognition. Proceedings of the National Academy of Sciences, 103(2), 449-454.

Barbas, H. (1993). Organization of cortical afferent input to orbitofrontal areas in the rhesus monkey. Neuroscience, 56(4), 841-864.

Baxter, M. G., \& Murray, E. A. (2002). The amygdala and reward. Nature Reviews Neuroscience, 3(7), 563-573.

Brett, M., Leff, A. P., Rorden, C., \& Ashburner, J. (2001). Spatial normalization of brain images with focal lesions using cost function masking. Neuroimage, 14(2), 486-500.

Browning, P. G., Easton, A., \& Gaffan, D. (2007). Frontal-temporal disconnection abolishes object discrimination learning set in macaque monkeys. Cerebral Cortex, 17(4), 859-864.

Bussey, T. J., Saksida, L. M., \& Murray, E. A. (2002). Perirhinal cortex resolves feature ambiguity in complex visual discriminations. European Journal of Neuroscience, 15(2), 365-374.

Butter, C. (1969). Perseveration in extinction and in discrimination reversal following selective frontal ablations in Macaca mulatta. Physiology and Behaviour, 4, 163-171.

Chudasama, Y., \& Robbins, T. W. (2003). Dissociable contributions of the orbitofrontal and infralimbic cortex to pavlovian autoshaping and discrimination reversal learning: Further evidence for the functional heterogeneity of the rodent frontal cortex. Journal of Neuroscience, 23(25), 87718780.

Cools, R., Barker, R. A., Sahakian, B. J., \& Robbins, T. W. (2001). Enhanced or impaired cognitive function in Parkinson's disease as a function of dopaminergic medication and task demands. Cerebral Cortex, 11(12), 1136-1143.

Cools, R., Barker, R. A., Sahakian, B. J., \& Robbins, T. W. (2003). L-Dopa medication remediates cognitive inflexibility, but increases impulsivity in patients with Parkinson's disease. Neuropsychologia, 41(11), 1431-1441.

Cools, R., Lewis, S. J., Clark, L., Barker, R. A., \& Robbins, T. W. (2007). 1DOPA disrupts activity in the nucleus accumbens during reversal learning in Parkinson's disease. Neuropsychopharmacology, 32(1), 180-189.

Dias, R., Robbins, T. W., \& Roberts, A. C. (1996). Dissociation in prefrontal cortex of affective and attentional shifts. Nature, 380(6569), 69-72.

Ell, S. W., Marchant, N. L., \& Ivry, R. B. (2006). Focal putamen lesions impair learning in rule-based, but not information-integration categorization tasks. Neuropsychologia, 44(10), 1737-1751.
Fera, F., Weickert, T. W., Goldberg, T. E., Tessitore, A., Hariri, A., Das, S., et al. (2005). Neural mechanisms underlying probabilistic category learning in normal aging. Journal of Neuroscience, 25(49), 11340-11348.

Fletcher, P., Buchel, C., Josephs, O., Friston, K., \& Dolan, R. (1999). Learningrelated neuronal responses in prefrontal cortex studied with functional neuroimaging. Cerebral Cortex, 9(2), 168-178.

Frank, M. J. (2005). Dynamic dopamine modulation in the basal ganglia: A neurocomputational account of cognitive deficits in medicated and nonmedicated Parkinsonism. Journal of Cognitive Neuroscience, 17(1), 51-72.

Frank, M. J., \& Claus, E. D. (2006). Anatomy of a decision: Striato-orbitofrontal interactions in reinforcement learning, decision making, and reversal. Psychological Review, 113(2), 300-326.

Frey, S., \& Petrides, M. (2000). Orbitofrontal cortex: A key prefrontal region for encoding information. Proceedings of the National Academy of Sciences USA, 97(15), 8723-8727.

Gaffan, D., \& Murray, E. A. (1990). Amygdalar interaction with the mediodorsal nucleus of the thalamus and the ventromedial prefrontal cortex in stimulus-reward associative learning in the monkey. Journal of Neuroscience, 10(11), 3479-3493.

Gluck, M. A., \& Bower, G. H. (1988). From conditioning to category learning: An adaptive network model. Journal of Experimental Psychology: General, 117(3), 227-247.

Gluck, M. A., Shohamy, D., \& Myers, C. (2002). How do people solve the "weather prediction" task?: Individual variability in strategies for probabilistic category learning. Learning and Memory, 9(6), 408-418.

Harlow, H. (1949). The formation of learning sets. Psychological Review, 56 , $51-65$.

Harlow, H. F., Davis, R. T., Settlage, P. H., \& Meyer, D. R. (1952). Analysis of frontal and posterior association syndromes in brain-damaged monkeys. Journal of Comparative Physiology and Psychology, 45(5), 419-429.

Hay, J. F., Moscovitch, M., \& Levine, B. (2002). Dissociating habit and recollection: Evidence from Parkinson's disease, amnesia and focal lesion patients. Neuropsychologia, 40(8), 1324-1334.

Hopkins, R. O., Myers, C. E., Shohamy, D., Grossman, S., \& Gluck, M. (2004). Impaired probabilistic category learning in hypoxic subjects with hippocampal damage. Neuropsychologia, 42(4), 524-535.

Hornak, J., O'Doherty, J., Bramham, J., Rolls, E. T., Morris, R. G., Bullock, P. R., et al. (2004). Reward-related reversal learning after surgical excisions in orbito-frontal or dorsolateral prefrontal cortex in humans. Journal of Cognitive Neuroscience, 16(3), 463-478.

Iversen, S. D., \& Mishkin, M. (1970). Perseverative interference in monkeys following selective lesions of the inferior prefrontal convexity. Experimental Brain Research, 11(4), 376-386.

Johnson, S. C., Schmitz, T. W., Asthana, S., Gluck, M. A., \& Myers, C. E. (in press). Associative learning over trials activates the hippocampus in healthy elderly but not mild cognitive impairment. Aging Neuropsychology and Cognition. [Epub ahead of print].

Kehoe, E. J. (1988). A layered network model of associative learning: Learning to learn and configuration. Psychological Review, 95(4), 411-433.

Kish, S. J., Shannak, K., \& Hornykiewicz, O. (1988). Uneven pattern of dopamine loss in the striatum of patients with idiopathic Parkinson's disease. Pathophysiologic and clinical implications. New England Journal of Medicine, 318(14), 876-880.

Kincses, T. Z., Antal, A., Nitsche, M. A., Bartfai, O., \& Paulus, W. (2004). Facilitation of probabilistic classification learning by transcranial direct current stimulation of the prefrontal cortex in the human. Neuropsychologia, 42(1), 113-117.

Knowlton, B. J., Squire, L. R., \& Gluck, M. A. (1994). Probabilistic classification learning in amnesia. Learning and Memory, 1(2), 106-120.

Knowlton, B. J., Mangels, J. A., \& Squire, L. R. (1996). A neostriatal habit learning system in humans. Science, 273(5280), 1399-1402.

Knowlton, B. J., Squire, L. R., Paulsen, J. S., Swerdlow, N., Swenson, M., \& Butters, N. (1996). Dissociations within nondeclarative memory in Huntington's disease. Neuropsychology, 10, 1-11.

Kruschke, J. K. (1996). Dimensional relevance shifts in category learning. Connection Science, 8(2), 225-247.

Mackintosh, N. J. (1975). A Theory of attention: Variations in the associability of stimuli with reinforcement. Psychological Review, 82(4), 276-298. 
Maddox, W. T., \& Ashby, F. G. (2004). Dissociating explicit and procedurallearning based systems of perceptual category learning. Behavioural Processes, 66(3), 309-332.

Meeter, M., Myers, C. E., Shohamy, D., Hopkins, R. O., \& Gluck, M. A (2006). Strategies in probabilistic categorization: Results from a new way of analyzing performance. Learning and Memory, 13(2), 230-239.

Meunier, M., Bachevalier, J., \& Mishkin, M. (1997). Effects of orbital frontal and anterior cingulate lesions on object and spatial memory in rhesus monkeys. Neuropsychologia, 35(7), 999-1015.

Murray, E. A., \& Gaffan, D. (2006). Prospective memory in the formation of learning sets by rhesus monkeys (Macaca mulatta). Journal of Experimental Psychology: Animal Behavior Processes, 32(1), 87-90.

Myers, C. E., Kluger, A., Golomb, J., Ferris, S., de Leon, M. J., Schnirman, G., et al. (2002). Hippocampal atrophy disrupts transfer generalization in nondemented elderly. Journal of Geriatric Psychiatry and Neurology, 15(2), 8290.

Nelson, H., \& Willison, J. (1991). National adult reading test (NART) test manual. Windsor, UK: NFER-Nelson.

Owen, A. M., James, M., Leigh, P. N., Summers, B. A., Marsden, C. D., Quinn, N. P., et al. (1992). Fronto-striatal cognitive deficits at different stages of Parkinson's disease. Brain, 115(Pt 6), 1727-1751.

Parker, A., \& Gaffan, D. (1998). Memory after frontal/temporal disconnection in monkeys: Conditional and non-conditional tasks, unilateral and bilateral frontal lesions. Neuropsychologia, 36(3), 259-271.

Passingham, R. E. (1993). The frontal lobes and voluntary action. Oxford: Oxford University Press.

Pasupathy, A., \& Miller, E. K. (2005). Different time courses of learningrelated activity in the prefrontal cortex and striatum. Nature, 433(7028), 873876.

Petrides, M. (1985). Deficits on conditional associative-learning tasks after frontal- and temporal-lobe lesions in man. Neuropsychologia, 23(5), 601-614.

Petrides, M. (1990). Nonspatial conditional learning impaired in patients with unilateral frontal but not unilateral temporal lobe excisions. Neuropsychologia, 28(2), 137-149.

Petrides, M. (1997). Visuo-motor conditional associative learning after frontal and temporal lesions in the human brain. Neuropsychologia, 35(7), 989997.

Petrides, M., \& Milner, B. (1982). Deficits on subject-ordered tasks after frontaland temporal-lobe lesions in man. Neuropsychologia, 20(3), 249-262.

Poldrack, R. A., Prabhakaran, V., Seger, C. A., \& Gabrieli, J. D. (1999). Striatal activation during acquisition of a cognitive skill. Neuropsychology, 13(4), 564-574.

Roesch, M. R., \& Olson, C. R. (2004). Neuronal activity related to reward value and motivation in primate frontal cortex. Science, 304(5668), 307-310.

Rolls, E. T. (2000). The orbitofrontal cortex and reward. Cerebral Cortex, 10(3), 284-294.
Rorden, C., \& Brett, M. (2000). Stereotaxic display of brain lesions. Behavioural Neurology, 12(4), 191-200.

Schoenbaum, G., Setlow, B., \& Ramus, S. J. (2003). A systems approach to orbitofrontal cortex function: Recordings in rat orbitofrontal cortex reveal interactions with different learning systems. Behavioural Brain Research, 146(1-2), 19-29.

Seger, C. A., \& Cincotta, C. M. (2005). The roles of the caudate nucleus in human classification learning. Journal of Neuroscience, 25(11), 2941-2951.

Shanks, D. R. (1995). Psychology of associative learning. Cambridge, England: Cambridge University Press.

Shohamy, D., Myers, C. E., Grossman, S., Sage, J., Gluck, M. A., \& Poldrack, R. A. (2004). Cortico-striatal contributions to feedback-based learning: Converging data from neuroimaging and neuropsychology. Brain, 127( $\mathrm{Pt} 4)$, 851-859.

Shohamy, D., Myers, C. E., Onlaor, S., \& Gluck, M. A. (2004). Role of the basal ganglia in category learning: How do patients with Parkinson's disease learn? Behavioural Neuroscience, 118(4), 676-686.

Shohamy, D., Myers, C. E., Geghman, K. D., Sage, J., \& Gluck, M. A. (2006). L-Dopa impairs learning, but spares generalization, in Parkinson's disease. Neuropsychologia, 44(5), 774-784.

Swainson, R., Rogers, R. D., Sahakian, B. J., Summers, B. A., Polkey, C. E., \& Robbins, T. W. (2000). Probabilistic learning and reversal deficits in patients with Parkinson's disease or frontal or temporal lobe lesions: Possible adverse effects of dopaminergic medication. Neuropsychologia, 38(5), 596-612.

Swainson, R., SenGupta, D., Shetty, T., Watkins, L. H., Summers, B. A., Sahakian, B. J., et al. (2006). Impaired dimensional selection but intact use of reward feedback during visual discrimination learning in Parkinson's disease. Neuropsychologia, 44(8), 1290-1304.

Tremblay, L., \& Schultz, W. (1999). Relative reward preference in primate orbitofrontal cortex. Nature, 398(6729), 704-708.

Tzourio-Mazoyer, N., Landeau, B., Papathanassiou, D., Crivello, F., Etard, O., Delcroix, N., et al. (2002). Automated anatomical labeling of activations in SPM using a macroscopic anatomical parcellation of the MNI MRI singlesubject brain. Neuroimage, 15(1), 273-289.

Wallis, J. D. (2007). Orbitofrontal cortex and its contribution to decision-making. Annual Review of Neuroscience, 30, 31-56.

Wallis, J. D., \& Miller, E. K. (2003). Neuronal activity in primate dorsolateral and orbital prefrontal cortex during performance of a reward preference task. European Journal of Neuroscience, 18(7), 2069-2081.

Webster, M. J., Bachevalier, J., \& Ungerleider, L. G. (1994). Connections of inferior temporal areas TEO and TE with parietal and frontal cortex in macaque monkeys. Cerebral Cortex, 4(5), 470-483

Yin, H. H., \& Knowlton, B. J. (2006). The role of the basal ganglia in habit formation. Nature Reviews Neuroscience, 7(6), 464-476.

Yokoyama, C., Tsukada, H., Watanabe, Y., \& Onoe, H. (2005). A dynamic shift of neural network activity before and after learning-set formation. Cerebral Cortex, 15(6), 796-801. 